\title{
QCD AT FINITE DENSITY
}

\author{
XIANG-QIAN LUO, ERIC B. GREGORY, SHUO-HONG GUO \\ Department of Physics, Zhongshan University Guangzhou 510275, China \\ HELMUT KRÖGER \\ Département de Physique, Université Laval, Québec, Québec G1K 7P4, Canada
}

\begin{abstract}
At sufficiently high temperature and density, quantum chromodynamics (QCD) predicts phase transition from the hadronic phase to the quark-gluon plasma phase. Lattice QCD is the most useful tool to investigate this critical phenomenon, which status is briefly reviewed. The usual problem in the Lagrangian formulation at finite density is either an incorrect continuum limit or its complex action and a premature onset of the transition as the chemical potential is raised. We show how the difficulties are overcome in our Hamiltonian approach.
\end{abstract}

\section{Introduction}

\subsection{Motivation}

According to the standard model of cosmology, 12-15 billion years ago, the early universe underwent a series of drastic changes. Several microseconds after the big bang it was in a hot and dense quark-gluon plasma (QGP) state, where quarks and gluons were deconfined. Several minutes later, it changed to a phase, with the quarks and gluons confined inside the hadrons. Today it remains in the low temperature and low density hadronic phase. The ultimate goal of machines such as the Relativistic Heavy Ion Collider (RHIC) at BNL and the Large Hadron Collider (LHC) at CERN is to create the high temperature QGP phase and replay the birth and evolution of the universe. In February 2000, physicists at CERN declared that they had experimentally

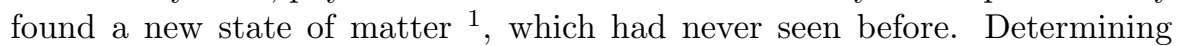
whether it is a QGP state requires further investigation from the future RHIC or LHC experiments. QGP may also exist at low temperature, in the core of very dense stars such as neutron stars.

Quantum chromodynamics (QCD) is the fundamental theory of quarks and gluons. Figure 1 shows a recently proposed 1 two-flavor QCD phase diagram on the temperature $T$ and chemical potential $\mu$ plane. It assembles the information accumulated from lattice QCD, random matrix model, and other approximation methods. There is confinement and spontaneous chiralsymmetry breaking in the hadronic phase, which is separated from other phases by a chiral phase transition. The transition changes from second to 
first order at a tricritical point. At high densities and low temperatures, a superconducting phase "2SC" was recently found in which up and down quarks with two out of three colors form Cooper-pairs characterized by a diquark condensate $\langle\psi \psi\rangle$. The transition between this 2SC phase and the QGP phase is likely first order. The chiral phase transition on the horizontal axis between the hadronic and 2SC phases is first order.

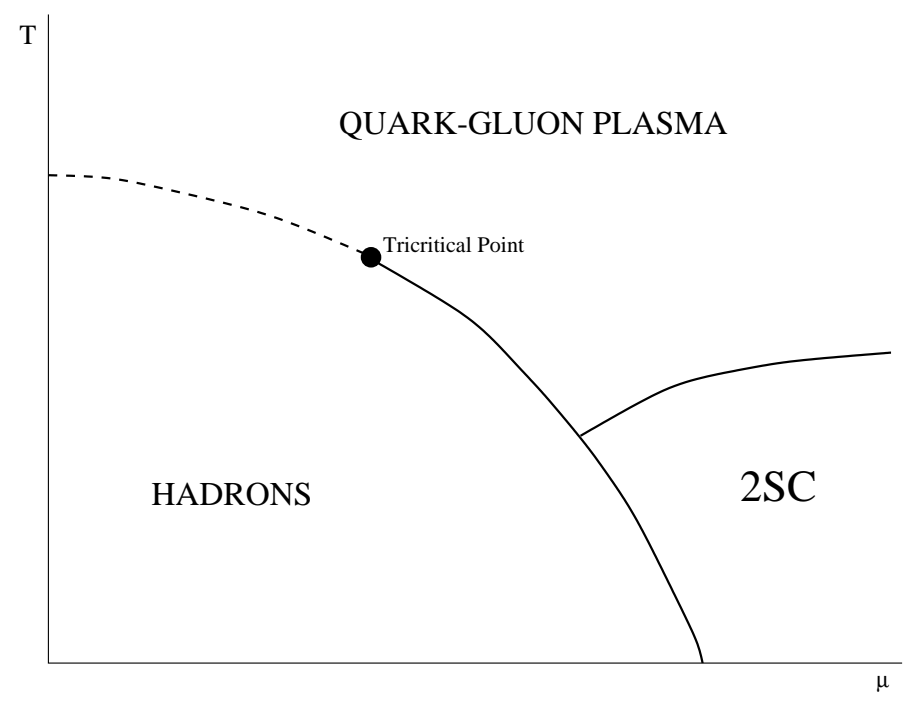

Figure 1. Phase diagram of QCD with 2 flavors.

A precise understanding of the QCD phase structure will provide valuable information in the experimental search for the QGP. Lattice gauge theory (LGT) proposed by Wilson in 1974, is a first principle nonperturbative technique for the investigation of phase transitions. There are no free parameters in LGT when the continuum limit is taken, in contrast with other nonperturbative techniques. Although the standard lattice Lagrangian Monte Carlo method works very well for QCD at finite temperature, it unfortunately breaks down at non-zero chemical potential (due to the so-called complex action problem). This is briefly reviewed in Sect. 1.2. In Sect. 2, we present our recently developed Hamiltonian approach 3 to lattice QCD at finite density, by introducing the chemical potential in a natural way, which avoids those usual problems. 


\subsection{Present status}

In the continuum, the grand canonical partition function of QCD at finite temperature $T$ and chemical potential $\mu$ is given by

$$
Z=\operatorname{Tr} \mathrm{e}^{-\beta(\mathrm{H}-\mu \mathrm{N})}, \quad \beta=\left(\mathrm{k}_{\mathrm{B}} \mathrm{T}\right)^{-1},
$$

where $k_{B}$ is the Boltzmann constant, $H$ is the Hamiltonian, and $N$ is particle number operator

$$
N=\int d^{3} x \psi^{\dagger}(x) \psi(x) .
$$

The energy density of the system with free quarks is given by $\$$

$$
\epsilon=\frac{1}{V} \frac{1}{Z} \operatorname{Tr} \mathrm{H}^{-\beta(\mathrm{H}-\mu \mathrm{N})}=-\left.\frac{1}{\mathrm{~V}} \frac{\partial \ln \mathrm{Z}}{\partial \beta}\right|_{\mu \beta} .
$$

Going over to $T \rightarrow 0$ and chiral limit $m \rightarrow 0$, one gets the energy density (where the contribution of $\mu=0$ is subtracted)

$$
\epsilon_{\text {sub }}=\frac{\mu^{4}}{4 \pi^{2}} .
$$

Unfortunately, in Lagrangian LGT, a naive discretization of the chemical potential term does not lead to the correct continuum relation Eq.(位). Let us take the naive fermions as an example. The action reads

$$
S_{f}=a^{4} \sum_{x} m \bar{\psi}(x) \psi(x)+\frac{a^{3}}{2} \sum_{x, k} \bar{\psi}(x) \gamma_{k} \psi(x+\hat{k})+a^{4} \mu \sum_{x} \psi^{\dagger}(x) \psi(x),
$$

where $\gamma_{-k}=-\gamma_{k}$. In the chiral limit $m \rightarrow 0$ and the continuum limit $a \rightarrow 0$, the subtracted energy density from this action is $\epsilon_{s u b} \propto(\mu / a)^{2}$, i.e. becoming quadratically divergent $\mathrm{E}$, and therefore it is inconsistent with the continuum result of Eq.(1). This problem is not due to the species doubling of naive fermions, because the case of Kogut-Susskind fermions or Wilson fermions is similar.

Hasenfratz and Karsch 5 proposed to introducing the chemical potential exponentially

$$
\begin{aligned}
S_{f} & =a^{4} \sum_{x} m \bar{\psi}(x) \psi(x)+\frac{a^{3}}{2} \sum_{x} \sum_{j=1}^{3}\left[\bar{\psi}(x) \gamma_{j} \psi(x+\hat{j})-\bar{\psi}(x+\hat{j}) \gamma_{j} \psi(x)\right] \\
& +\frac{a^{3}}{2} \sum_{x}\left[e^{\mu a} \bar{\psi}(x) \gamma_{4} \psi(x+\hat{4})-e^{-\mu a} \bar{\psi}(x+\hat{4}) \gamma_{4} \psi(x)\right] .
\end{aligned}
$$


The chemical potential can be introduced analogously for KS as well as for Wilson fermions. Such treatment of the chemical potential is numerically feasible in the quenched approximation (where the fermionic determinant det $\Delta$ is constraint to be 1, and quark loops are suppressed). However, there is evidence 6 that the quenched approximation produces an unphysical onset of the critical chemical potential at the value $\mu_{C}=M_{\pi}(m \neq 0) / 2$, which goes to zero in the chiral limit $\left[M_{\pi}(m \neq 0)\right.$ is the pion mass at finite bare quark mass $m$. A finite bare quark mass has to be introduced in most of the numerical simulations]. This is in conflict with other theoretical predictions $\mu_{C} \approx M_{N}^{(0)} / 3\left[M_{N}^{(0)}\right.$ is the nucleon mass at $\left.\mu=0\right]$.

For full QCD, the fermionic degrees of freedom have to be integrated out, and in the measure occurs $\operatorname{det} \Delta$. For finite chemical potential $\operatorname{det} \Delta$ from Eq. (6) and the effective fermionic action $\ln \operatorname{det} \Delta$ become complex, due to $\Delta_{y, x} \neq \gamma_{5} \Delta_{x, y}^{\dagger} \gamma_{5}$, which renders numerical simulations extremely difficult. Much effort has been made to solve this notorious complex action problem:

(1) The Glasgow group has suggested to treat $\operatorname{det} \Delta$ as observabled. This method requires a very large number of configurations, in particular for $\mu \approx$ $\mu_{C}$. Even on a very small laftice $V=4^{4}$, the computational costs exceed the current computer capacity 6 . Even in this case, the unphysical onset of $\mu_{C}$ still exists. Therefore, it is unclear whether the onset is an intrinsic problem of the proposal Eq. (6).

(2) In the imaginary chemical potential method $9 \operatorname{det} \Delta$ becomes real, which works well for numerical simulations at high temperature and low density. But it might not work at low temperature and high density.

(3) It has been proposed to utilize a special symmetry 10 . This is the only syccessful method in Lagrangian formulation, and attracts much attention 11.12, but it works only for the $\mathrm{SU}(2)$ gauge group.

(4) In 11 , the probability distribution function method 13 is applied to 2-color QCD (free of complex action problem), and very interesting results for the diquark condensate $\langle\psi \psi\rangle$ are obtained.

\section{Hamiltonian Approach}

\subsection{Free fermions at zero chemical potential}

The lattice Hamiltonian describing noninteracting Wilson fermions in $d+1$ dimensions at $\mu=0$ is

$$
H=\sum_{x} m \bar{\psi}(x) \psi(x)+\sum_{x, k} \frac{1}{2 a} \bar{\psi}(x)\left(\gamma_{k} \psi(x+\hat{k})+r(\psi(x)-\psi(x+\hat{k}))\right) .
$$


The up and down components of ${ }^{2}$ are coupled via the $\gamma_{k}$ matrices, and one can use a unitary transformation 14

$$
H^{\prime}=\exp (-i S) H \exp (i S)
$$

to decouple them. The operator $S$ can be computed explicitly 14 in momentum space:

$$
S=-\sum_{p} \frac{\theta_{p}}{A_{p}} \sum_{j=1}^{d} \psi_{p}^{\dagger} \gamma_{j} \psi_{p} \frac{\sin p_{j} a}{a}, \quad A_{p}=\left(\sum_{j=1}^{d}\left(\frac{\sin p_{j} a}{a}\right)^{2}\right)^{1 / 2} .
$$

The physical vacuum state of $H$ reads

$$
|\Omega\rangle=\exp (i S)|0\rangle,
$$

where $\mid 0>$ is the bare vacuum state defined as $\xi|0\rangle=\eta|0\rangle=0$. The parameter $\theta_{p}$ in Eq. (9) is so determined that the vacuum energy $E_{\Omega}=\langle\Omega|H| \Omega\rangle=$ $\left\langle 0\left|H^{\prime}\right| 0\right\rangle$ is minimized. After the unitary transformation, the fermionic field $\psi$ can be simply expressed by up and down 2-spinors $\xi$ and $\eta^{\dagger}$ as $\psi=\left(\begin{array}{c}\xi \\ \eta^{\dagger}\end{array}\right)$ and $H^{\prime}$ is diagonal

$$
H^{\prime}=\sum_{p} A_{p}^{\prime} \bar{\psi}_{p} \psi_{p}=\sum_{p}\left(\left(m+\frac{2 r}{a} \sum_{j=1}^{d} \sin ^{2}\left(p_{j} a / 2\right)\right)^{2}+A_{p}^{2}\right)^{1 / 2} \bar{\psi}_{p} \psi_{p} .(11)
$$

For Wilson fermions, in the continuum limit $a \rightarrow 0$, for any finite momentum $p$, we have $E_{\Omega}=-2 N_{c} N_{f} \sum_{p} \sqrt{m^{2}+p^{2}}$, giving the correct dispersion relation. Here $N_{c}$ and $N_{f}$, respectively, are the number of colors and number of flavors.

\subsection{Free fermions at nonzero chemical potential}

Now we naturally introduce the chemical potential

$$
H_{\mu}=H-\mu N
$$

where $H$ is given by Eq.(7) and $N$ is given by Eq.(2). Let us define the state $\left|n_{p}, \bar{n}_{p}\right\rangle$ by

$$
\begin{array}{ll}
\xi_{p}\left|0_{p}, \bar{n}_{p}\right\rangle=0, & \xi_{p}^{\dagger}\left|0_{p}, \bar{n}_{p}\right\rangle=\left|1_{p}, \bar{n}_{p}\right\rangle, \quad \xi_{p}\left|1_{p}, \bar{n}_{p}\right\rangle=\left|0_{p}, \bar{n}_{p}\right\rangle, \quad \xi_{p}^{\dagger}\left|1_{p}, \bar{n}_{p}\right\rangle=0, \\
\eta_{p}\left|n_{p}, 0_{p}\right\rangle=0, & \eta_{p}^{\dagger}\left|n_{p}, 0_{p}\right\rangle=\left|n_{p}, 1_{p}\right\rangle, \quad \eta_{p}\left|n_{p}, 1_{p}\right\rangle=\left|n_{p}, 0_{p}\right\rangle, \quad \eta_{p}^{\dagger}\left|n_{p}, 1_{p}\right\rangle=0 .
\end{array}
$$

The numbers $n_{p}$ and $\bar{n}_{p}$ take the values 0 or 1 due to the Pauli principle. By definition, the up and down components of the fermion field are decoupled. 
Obviously, this is not an eigenstate of $H_{\mu}$ due to the non-diagonal form of $H$. However, they are eigenstates of $H_{\mu}^{\prime}$, which are related to $H_{\mu}$ by a unitary transformation

$$
H_{\mu}^{\prime}=\exp (-i S) H_{\mu} \exp (i S)=H^{\prime}-\mu N .
$$

For the vacuum eigenstate of $H_{\mu}$ we make an ansatz of the following form

$$
|\Omega\rangle=\exp (i S) \sum_{p} f_{n_{p}, \bar{n}_{p}}\left|n_{p}, \bar{n}_{p}\right\rangle .
$$

$S$ is given by Eq.(9), and $H^{\prime}$ is given by Eq. (11). The vacuum energy is $E_{\Omega}=2 N_{c} N_{f} \sum_{p} C_{n_{p}, \bar{n}_{p}}\left[\left(A_{p}^{\prime}-\mu\right) n_{p}+\left(A_{p}^{\prime}+\mu\right) \bar{n}_{p}-A_{p}^{\prime}-\mu\right]$. Here we have introduced the notation $C_{n_{p}, \bar{n}_{p}}=f_{n_{p}, \bar{n}_{p}}^{2}$, which have not yet been specified. For this purpose we use the stability condition of the vacuum. Because $\mu>0$, the vacuum energy increases with $n_{p}$. This means the vacuum is unstable unless $\bar{n}_{p}=0$. This simplifies the vacuum energy to $E_{\Omega}=2 N_{c} N_{f} \sum_{p}\left[C_{1_{p}}\left(A_{p}^{\prime}-\mu\right)-A_{p}^{\prime}-\mu\right]$, where we use the abbreviation $C_{n_{p}}=C_{n_{p}, 0}$ and the normalization condition. The dependence of $C_{1_{p}}$ on the value of $\mu$ can be seen by inspection of the derivative $\partial E_{\Omega} / \partial C_{1_{p}}=$ $2 N_{c} N_{f}\left(A_{p}^{\prime}-\mu\right)$. For $\mu>A_{p}^{\prime}$, the right-hand side is negative. Maximizing $C_{1_{p}}$ means minimizing the vacuum energy. Therefore, $C_{1_{p}}=1$. On the other hand, for $\mu<A_{p}^{\prime}$, the right-hand side is positive and for any $C_{1_{p}}$ the vacuum is unstable. Therefore, $C_{1_{p}}=0$. We can summarize these properties by writing $C_{1_{p}}=\Theta\left(\mu-A_{p}^{\prime}\right)$. Thus the vacuum expectation of $H$ becomes $\langle\Omega|H| \Omega\rangle=2 N_{c} N_{f} \sum_{p}\left(C_{1_{p}} A_{p}^{\prime}-A_{p}^{\prime}\right)$. The subtracted energy density reads

$$
\epsilon_{\text {sub }}=\frac{\langle\Omega|H| \Omega\rangle-\left.\langle\Omega|H| \Omega\rangle\right|_{\mu=0}}{N_{c} N_{f} N_{s}}=\frac{\mu^{4}}{4 \pi^{2}} .
$$

Here $N_{s}$ is the number of spatial lattice sites. Thus we have proven that our Hamiltonian approach to free quarks at finite chemical potential leads to the correct continuum result for the vacuum energy density, Eq. (何). From this relation, we can easily see that the free quark number density is proportional to $\mu^{3}$. For naive fermions, in the continuum limit $a=0$, there will be an extra factor of $2^{d}$.

\subsection{Strong coupling $Q C D$ at nonzero chemical potential}

As is well known, lattice QCD at $\mu=0$ confines quarks and spontaneously breaks chiral symmetry. For a sufficiently large chemical potential, this picture

$\overline{a^{a} \text { There is a typo in Eqs. (2.24) and }}(2.25)$ in $\mathrm{l}$ : $E_{\Omega}$ there should be read as $\langle\Omega|H| \Omega\rangle$. The results and conlusions are the same. 


\begin{tabular}{|c|c|c|c|c|c|c|c|c|}
\hline$\Gamma_{A}$ & 1 & $\gamma_{j}$ & $\gamma_{4}$ & $\gamma_{5}$ & $i \gamma_{4} \gamma_{5}$ & $i \gamma_{4} \gamma_{j}$ & $i \epsilon_{j j_{1} j_{2}} \gamma_{j_{1}} \gamma_{j_{2}}$ & $i \epsilon_{j j_{1} j_{2}} \gamma_{4} \gamma_{j_{1}} \gamma_{j_{2}}$ \\
\hline$L_{A}$ & 1 & $2 \delta_{k, j}-1$ & -1 & -1 & 1 & $1-2 \delta_{k, j}$ & $2 \delta_{k, j}-1$ & $1-2 \delta_{k, j}$ \\
\hline
\end{tabular}

Table 1. $\Gamma$ matrices and coefficients.

may change. Here we set out to investigate finite density QCD in the strong coupling regime $1 / g^{2}<<1$. Following reference 14, $H^{\prime}$ in Eq. (13) is now replaced by

$$
\begin{aligned}
H^{\prime} & =\left[m\left[1-\left(2 \theta_{0}\right)^{2} d\right]+\frac{\left(2 \theta_{0}\right) d}{a}\right] \sum_{x} \bar{\psi}(x) \psi(x)+\frac{g^{2} C_{N} d\left(2 \theta_{0}\right)^{2}}{4 a} \sum_{x} \psi^{\dagger}(x) \psi(x) \\
& -\frac{g^{2} C_{N}\left(2 \theta_{0}\right)^{2}}{32 a N_{c}} \sum_{x, k} L_{A} \psi_{f_{1}}^{\dagger}(x) \Gamma_{A} \psi_{f_{2}}(x) \psi_{f_{2}}^{\dagger}(x+k) \Gamma_{A} \psi_{f_{1}}(x+k)
\end{aligned}
$$

The additional four-fermion term is induced by gauge interactions with fermions. There $d=3$ denotes the spatial dimension, $f_{1}, f_{2}$ are flavor indices (summation over repeated indices is understood), $\theta_{0}=1 /\left(4 m a+g^{2} C_{N}\right)$, and $C_{N}=\left(N_{c}^{2}-1\right) /\left(2 N_{c}\right)$. The matrices $\Gamma_{A}$ and their coefficients $L_{A}$ are given in Tab.[1].

The unitary transformed Hamiltonian Eq. (16) can also be re-expressed in terms of the following pseudoscalar and vector operators 14

$$
\begin{aligned}
& \Pi=\frac{1}{2 \sqrt{-\bar{v}}} \psi^{\dagger}\left(1-\gamma_{4}\right) \gamma_{5} \psi, \\
& V_{j}=\frac{1}{2 \sqrt{-\bar{v}}} \psi^{\dagger}\left(1-\gamma_{4}\right) \gamma_{j} \psi,
\end{aligned}
$$

as 目

$$
\begin{aligned}
H^{\prime} & =E_{\Omega}^{(0)}+G_{1} \sum_{p}\left(\Pi^{\dagger}(p) \Pi(p)+\sum_{j} V_{j}^{\dagger}(p) V_{j}(p)\right) \\
& +G_{2} \sum_{p}\left(\Pi^{\dagger}(p) \Pi^{\dagger}(-p)+\text { h.c. }\right) \sum_{j} \cos p_{j} a \\
& +G_{2} \sum_{p, j}\left(V_{j}^{\dagger}(p) V_{j}^{\dagger}(-p)+\text { h.c. }\right)\left(\sum_{j^{\prime}} \cos p_{j^{\prime}} a-2 \cos p_{j} a\right),
\end{aligned}
$$

where

$$
E_{\Omega}^{(0)}=N_{f} N_{s}\left[m\left[1-\left(2 \theta_{0}\right)^{2} d\right]+\frac{\left(2 \theta_{0}\right) d}{a}\right] \bar{v}
$$




$$
\begin{aligned}
& +N_{f} N_{s} \frac{g^{2} C_{N} d\left(2 \theta_{0}\right)^{2}}{4 a} v^{\dagger}-N_{f} N_{s} \frac{g^{2} C_{N}\left(2 \theta_{0}\right)^{2} d}{16 a N_{c}}\left(v_{2}^{\dagger}-\bar{v}_{2}\right), \\
G_{1} & =2\left[m\left[1-\left(2 \theta_{0}\right)^{2} d\right]+\frac{\left(2 \theta_{0}\right) d}{a}\right]+\frac{g^{2} C_{N} d\left(2 \theta_{0}\right)^{2}}{4 a N_{c}} \bar{v}, \\
G_{2} & =-\frac{g^{2} C_{N}\left(2 \theta_{0}\right)^{2}}{8 a N_{c}} \bar{v}, \\
v^{\dagger} & =\frac{2 N_{c}}{N_{s}} \sum_{p} C_{n_{p}, \bar{n}_{p}}\left(n_{p}-\bar{n}_{p}+1\right), \\
\bar{v} & =\frac{2 N_{c}}{N_{s}} \sum_{p} C_{n_{p}, \bar{n}_{p}}\left(n_{p}+\bar{n}_{p}-1\right), \\
v_{2}^{\dagger} & =\frac{\left(2 N_{c}\right)^{2}}{N_{s}} \sum_{p} C_{n_{p}, \bar{n}_{p}}\left(n_{p}-\bar{n}_{p}+1\right)^{2}, \\
\bar{v}_{2} & =\frac{\left(2 N_{c}\right)^{2}}{N_{s}} \sum_{p} C_{n_{p}, \bar{n}_{p}}\left(n_{p}+\bar{n}_{p}-1\right)^{2} .
\end{aligned}
$$

Eq. (18) can be diagonalized by a Bogoliubov transformation 14

$$
\begin{gathered}
P(p)=\cosh u(p) a(p)+\sinh u(p) a^{\dagger}(-p), \\
V_{j}(p)=\cosh v_{j}(p) b(p)+\sinh v_{j}(p) b^{\dagger}(-p), \\
\tanh 2 u(p)=\frac{-2 G_{2}}{G_{1}} \sum_{j} \cos p_{j} a, \\
\tanh 2 v_{j}(p)=\frac{-2 G_{2}}{G_{1}}\left(\sum_{j^{\prime}} \cos p_{j^{\prime}} a-2 \cos p_{j} a\right) .
\end{gathered}
$$

The Bogoliubov transformed Hamiltonian eventually becomes

$$
\begin{aligned}
H^{\prime \prime} & =E_{\Omega}^{(0)}+\frac{N_{f}^{2}}{2} G_{1} \sum_{p}\left[\sqrt{1-\tanh ^{2} 2 u(p)}-1\right] \\
& +\frac{N_{f}^{2}}{2} G_{1} \sum_{p, j}\left[\sqrt{1-\tanh ^{2} 2 v_{j}(p)}-1\right] \\
& +G_{1} \sum_{p} \sqrt{1-\tanh ^{2} 2 u(p)} a^{\dagger}(p) a(p) \\
& +G_{1} \sum_{p, j} \sqrt{1-\tanh ^{2} 2 v_{j}(p)} b_{j}^{\dagger}(p) b_{j}(p) .
\end{aligned}
$$


Using the notation, normalization condition and arguments for the coefficient as in Sect. 2.2, we obtain $\bar{n}_{p}=0$ and $C_{1_{p}}=\Theta\left(\mu-m_{d y n}^{(0)}\right)$. Here $m_{d y n}^{(0)}=$ $d /\left(a g^{2} C_{N}\right)$ is the dynamical quark mass at $\mu=0$. Therefore, in the chiral limit $m=0$, the vacuum energy is

$$
\begin{aligned}
\frac{E_{\Omega}}{2 N_{c} N_{f} N_{s}} & =\left(m_{d y n}^{(0)}-\mu\right) \Theta\left(\mu-m_{d y n}^{(0)}\right)-m_{d y n}^{(0)}-\mu \\
& +\frac{N_{f}}{2 N_{s}} G_{1} \sum_{p}\left[\sqrt{1-\tanh ^{2} 2 u(p)}-1\right] \\
& +\frac{N_{f}}{2 N_{s}} G_{1} \sum_{p, j}\left[\sqrt{1-\tanh ^{2} 2 v_{j}(p)}-1\right] .
\end{aligned}
$$

According to the Feynman-Hellmann theorem, the chiral condensate is related to the ground state energy by

$$
\langle\bar{\psi} \psi\rangle=\frac{1}{N_{f} N_{s}} \lim _{m \rightarrow 0} \frac{\partial E_{\Omega}(m \neq 0)}{\partial m}=\langle\bar{\psi} \psi\rangle^{(0)}\left[1-\Theta\left(\mu-m_{d y n}^{(0)}\right)\right],
$$

where $\langle\bar{\psi} \psi\rangle^{(0)}$ is the chiral condensate at $\mu=0$

$$
\langle\bar{\psi} \psi\rangle^{(0)}=-2 N_{C}\left(1-\frac{4 d}{g^{4} C_{N}^{2}}\right)\left(1-\frac{N_{f}}{N_{c}} I_{1}-\frac{N_{f}}{N_{c}} I_{2}\right)
$$

and for $d=3, I_{1}=0.078354 \pm 2 \times 10^{-6}, I_{2}=0.235075 \pm 4 \times 10^{-6}$. According to Eq. (21), for $\mu<m_{d y n}^{(0)},\langle\bar{\psi} \psi\rangle=\langle\bar{\psi} \psi\rangle^{(0)} \neq 0$, i.e., chiral symmetry is spontaneously broken. For $\mu>m_{d y n}^{(0)},\langle\bar{\psi} \psi\rangle=0$, i.e., chiral symmetry is restored. Therefore, there is a first order chiral phase transition and the critical value of $\mu$ is given by

$$
\mu_{C}=m_{d y n}^{(0)}=\frac{d}{g^{2} C_{N} a},
$$

The critical chemical potential $\mu_{C}$ is equal to the dynamical quark mass at $\mu=0$, which agrees with the result from an entirely different method 16 in Hamiltonian formulation. (The authors argued this was a second order phase transition, in contrast we clearly observe a first order transition). Our result is consistent with other theoretical predictions $\mu_{C} \approx M_{N}^{(0)} / 3$, because (see below) at $\mu=0$ holds $M_{N}^{(0)} \approx 3 m_{d y n}^{(0)}$.

We can compute now the quark number density in the chiral limit $m=0$, which yields

$$
n_{q}=\frac{-1}{2 N_{c} N_{f} N_{s}} \frac{\partial E_{\Omega}}{\partial \mu}-1=\frac{\left\langle\Omega\left|\sum_{x} \psi^{\dagger}(x) \psi(x)\right| \Omega\right\rangle}{2 N_{c} N_{f} N_{s}}-1=\Theta\left(\mu-\mu_{C}\right),
$$


This is consistent with the $\beta=0$ simulation results described in 17, and however, is different from the large $\mu$ behavior in the continuum (i.e. the StefannBoltzmann law $n_{q} \propto \mu^{3}$ for free quarks). It remains to be seen whether higher order $1 / g^{2}$ calculations will improve this behavior.

The quark number susceptibility, standing for the response of the quark number density to infinitesimal changes in $\mu$, is

$$
\chi_{q}=\frac{\partial n_{q}}{\partial \mu}=\delta\left(\mu-\mu_{C}\right)
$$

Finally, let us look at some implications on the thermal mass spectrum of the pseudoscalar meson, vector meson and nucleon. The thermal mass is defined by $M_{h}^{\star}=\langle h|H-\mu N| h\rangle-E_{\Omega}$. For the pseudoscalar meson, in the chiral limit $m=0$,

$$
M_{\pi}^{\star}=G_{1} \sqrt{1-\tanh ^{2} 2 u(p=0)}=\left\{\begin{array}{cl}
0 & \text { for } \mu<\mu_{\mathrm{C}}, \\
4 m_{d y n}^{(0)} & \text { for } \mu>\mu_{\mathrm{C}} .
\end{array}\right.
$$

Therefore, in the broken phase, the pseudoscalar is a Goldstone boson $\left(M_{\pi}^{\star} \propto\right.$

$\sqrt{m} \rightarrow 0$ ), and in the symmetric phase, it is no longer a Goldstone boson. For the vector meson,

$$
M_{V}^{\star}=G_{1} \sqrt{1-\tanh ^{2} 2 v_{j}(p=0)}=\left\{\begin{array}{cl}
M_{V}^{(0)} & \text { for } \mu<\mu_{\mathrm{C}} \\
4 m_{d y n}^{(0)} & \text { for } \mu>\mu_{\mathrm{C}} .
\end{array}\right.
$$

where $M_{V}^{(0)}=4 \sqrt{d-1} /\left(a g^{2} C_{N}\right)$ is the vector mass at $\mu=0$. Therefore, $\partial M / \partial \mu \propto \delta\left(\mu-\mu_{C}\right)$ for the pseudoscalar and vector mesons. To see the critical behavior at zero temperature, one should be very close to $\mu_{C}$. This behavior is consistent with that of the quark number density. To see whether the meson thermal masses depend on $\mu$, higher order $1 / g^{2}$ corrections must be included.

For the nucleon, we obtain the expected behavior

$$
M_{N}^{\star}=M_{N}^{(0)}-3 \mu
$$

for $\mu<\mu_{C}$, where $M_{N}^{(0)} \approx 3 m_{d y n}^{(0)}$. This leads to $M_{N}^{\star}=0$ at $\mu=\mu_{C}$.

\section{Outlook}

In this paper, we have developed a Hamiltonian approach to lattice QCD at finite density. The chemical potential is introduced in a very natural way as in the continuum. It avoids the persisting problem in the Lagrangian approach of an incorrect continuum limit, or complex action or a premature onset of 
the transition to nonzero quark density as $\mu$ is raised. The main result in the free case is given by Eq. (15), and those in the strong coupling regime are given by Eqs. (20)-(28). We have seen that the approach works well in the free case and also in the strong coupling regime. We predict that at strong coupling, the chiral transition is of first order, and the critical chemical potential $\mu_{C} \approx M_{N}^{(0)} / 3$.

We have not yet specified the nature of the chiral-symmetric phase for $\mu>\mu_{C}$. Is it a QGP phase or a color-superconduction phase 2 (see Fig. 1)? Up to now, there has been no first principle investigation of such a phase. Better understanding of it will affect our knowledge of the formation of the neutron star.

At this point, we should mention that the results of Hamiltonian formulation and Lagrangian formulation 18 might be quite different. It is interesting to see whether they describe the same physics in the continuum. For pure gauge theory, within a Hamiltonian approach, we can extend to the intermediate coupling and obtain meaningful results for the glueballs 19 . For fermions, the calculation is far from trivial. Recently we proposed a Monte Carlo technique in the Hamiltonian formulation 20 for the purpose to do nonperturbative numerical simulations, by combining the virtues of the Monte Carlo algorithm with importance sampling and the Hamiltonian approach. We hope to apply it to QCD and with the aim to obtain useful information for RHIC and LHC physics and neutron star phenomenology.

\section{Acknowledgments}

We are grateful to the attendees of the workshop for useful discussions. X.Q.L. is supported by the National Science Fund for Distinguished Young Scholars (19825117), National Natural Science Foundation (10010310687), the Ministry of Education, the Doctoral Program of Higher Education and Guangdong Provincial Natural Science Foundation (990212) of China. X.Q.L. and E.B.G. are supported by the Guangdong National Communication Ltd. H.K. has been supported by NSERC Canada.

\section{References}

1. http://press.web.cern.ch/.

2. K. Rajagopal, Nucl. Phys. A661, 150 (1999), and references there in.

3. E.B. Gregory, S. Guo, H. Kröger, X.Q. Luo, Phys. Rev. D62, 054508 (2000).

4. J.R. Li, Introduction to Quark Matter Theory, (Hunan Education Press, 1989); J. Kapusta, Finite Temperature Field Theory, (Cambridge Uni- 
versity Press, Cambridge, England, 1989); B. Freedman and L. Mclerran, Phys. Rev. D16, 1130, (1977); 161147 (1977); 161169 (1977).

5. P. Hasenfratz and F. Karsch, Phys. Lett. B125, 308 (1983).

6. J. Kogut, M.P. Lombardo, and D. Sinclair, Phys. Rev. D51, 1282 (1995); I. Barbour, S. Morrison, E. Klepfish, J. Kogut and M. Lombardo, Phys. Rev. D56, 7063 (1997).

7. I. Barbour, C. Davies, and Z. Sabeur, Phys. Lett. B215, 567 (1988).

8. R. Aloisio, V. Azcoiti, G. DiCarlo, A. Galante, and A. Grillo, Phys. Lett. B453, 275 (1999).

9. M. Alford, A. Kapustin and F. Wilczek, Phys. Rev. D59, 054502 (1999); M.P. Lombardo, Nucl. Phys. B(Proc. Suppl.)83. 375 (2000).

10. S. Hands, J. Kogut, M.P. Lombardo and S. Morrison, Nucl. Phys. B 558, 327 (1999).

11. R. Aloisio, A. Galante, V. Azcoiti, G. DiCarlo, and A. Grillo, these proceedings, hep-lat/0007018.

12. Y. Liu, O. Miyamura, A. Nakamura and T. Takaishi, hep-lat/0009009.

13. V. Azcoiti, V. Laliena, and X.Q. Luo, Phys. Lett. B354, 111 (1995).

14. X.Q. Luo and Q. Chen, Phys. Rev. D46 (1992) 814; Q. Chen, and X. Luo, ibid. 42, 1293 (1990).

15. J. Bjorken and S. Drell, Relativistic Quantum Mechanics (McGraw-Hill, New York); P. Roman, Advanced Quantum Theory (Addison-Wesley, New York).

16. A. Le Yaouanc, L. Oliver, O. Pene, J. Raynal, M. Jarfi and O. Lazrak, Phys. Rev. D37, 3691 (1988).

17. R. Aloisio, V. Azcoiti, G. DiCarlo, A. Galante and A. Grillo, Nucl. Phys. B564, 489 (2000).

18. N. Bilic, K. Demeterfi, B. Petersson, Nucl. Phys. B377, 651 (1992).

19. X.Q. Luo and Q. Chen, Mod. Phys. Lett. A11, 2435 (1996); X.Q. Luo, Q. Chen, S. Guo, X. Fang and J. Liu, Nucl. Phys. B(Proc. Suppl.)53, 243 (1997).

20. H. Jirari, H. Kröger, X.Q. Luo and K. Moriarty, Phys. Lett. A258, 6 (1999). X.Q. Luo, H. Jirari, H. Kröger, K. Moriarty, these proceedings. 